\title{
A case of a peculiarly shaped gastrointestinal
} stromal tumor
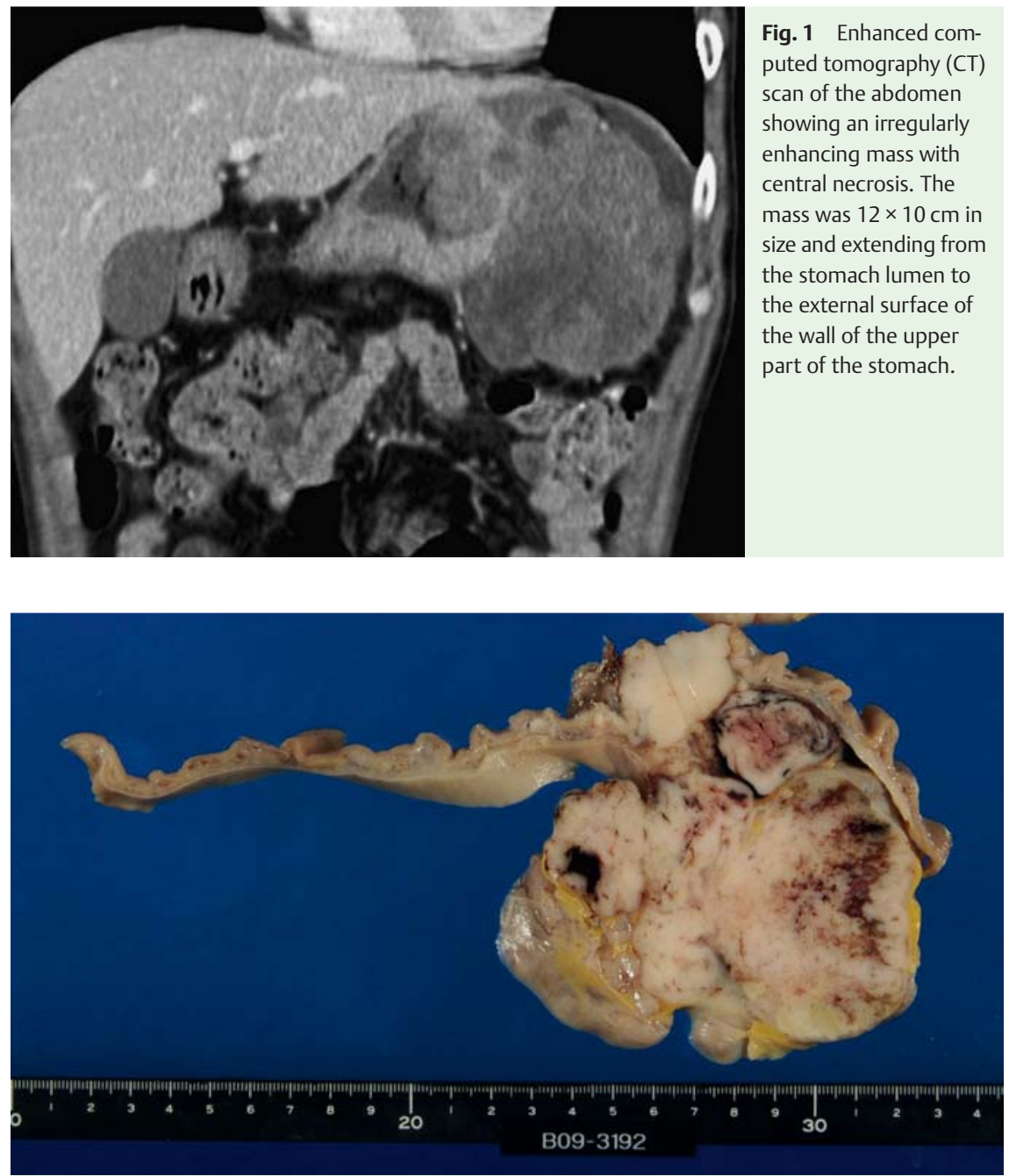

Fig. 3 Gross appearance of the resected specimen. Tumor was $14 \times 11 \times 10 \mathrm{~cm}$ with areas of necrosis and bleeding in the solid parenchyma.

A 67-year-old man presented at our hospital after having been diagnosed with severe anemia at a regular check-up. He had a history of tarry stool lasting for 1 month. Physical examination revealed abdominal distension with a firm palpable mass in the upper abdomen. Initial laboratory data showed severe anemia that needed emergency admission. An enhanced computed tomography (CT) scan revealed a $12 \times 10 \mathrm{~cm}$, irregularly enhancing mass with central necrosis extending from the stomach lumen to the external surface of the upper gastric wall ( $\bullet$ Fig. 1 ).

There was no nodal or distant metastasis evident on the CT scan. Gastrointestinal endoscopy revealed a snowman-shaped
Fig. 1 Enhanced computed tomography (CT) scan of the abdomen showing an irregularly enhancing mass with central necrosis. The mass was $12 \times 10 \mathrm{~cm}$ in size and extending from the stomach lumen to the external surface of the wall of the upper part of the stomach.

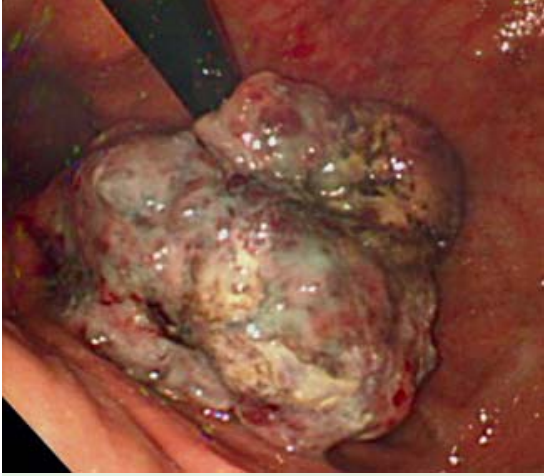

Fig. 2 Gastrointestinal endoscopy showed a snowman-shaped tumor, about $5 \mathrm{~cm}$ in diameter, located on the lesser curvature of the upper gastric body just below the gastric cardia.

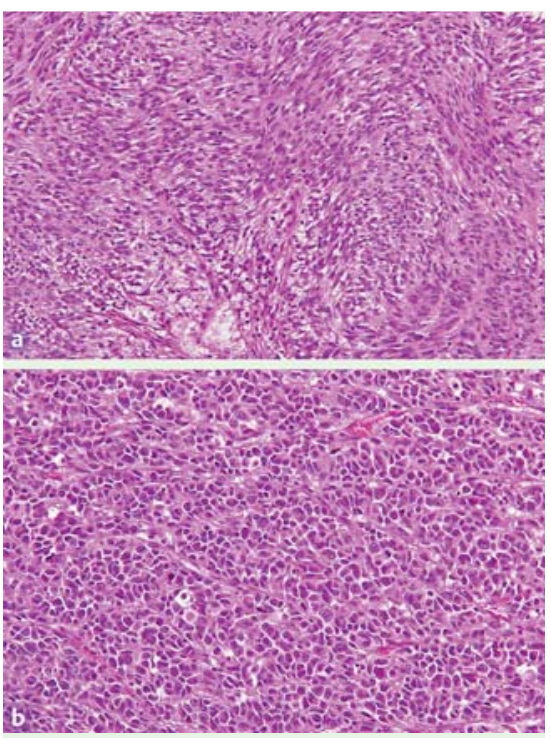

Fig. 4 Photomicrographs of the resected specimen showing: a spindle-shaped tumor cells $(90 \%)$; and $\mathbf{b}$ round cells $(10 \%)$ (Hematoxylin and eosin, magnification $\times 500)$.

about $5 \mathrm{~cm}$ in diameter, on the ser curvature of the upper gastric body just below the gastric cardia ( $\bullet$ Fig. 2 ).

The surface of the tumor was irregular, relatively soft, and bled easily. There were no ulcers or erosions.

Total gastrectomy was carried out and the tumor was completely resected. On gross examination the solid parenchyma of the tumor, which measured $14 \times 11 \times 10 \mathrm{~cm}$, showed focal areas of necrosis and bleeding ( $\bullet$ Fig. 3 ).

Microscopically the tumor consisted of spindle-shaped tumor cells (90\%) and round cells (10\%) ( Fig. 4), and no lymph node metastasis was found. Immunohistochemical studies revealed that the tu- mor cells were positive for c-kit, CD34, vimentin, and $\alpha$-smooth-muscle actin ( $\alpha$ SMA; partial), but negative for S-100 (๑ Fig. 5).

The labeling index (Ki-67) was about $50 \%$ $60 \%$. On the basis of these results, the tumor was diagnosed as a gastrointestinal stromal tumor(GIST) at high risk for malignancy.

Although GISTs are the most common mesenchymal tumors of the gastrointestinal tract [1], it is rare to find a snowmanshaped GIST, such as the one in the pres- 

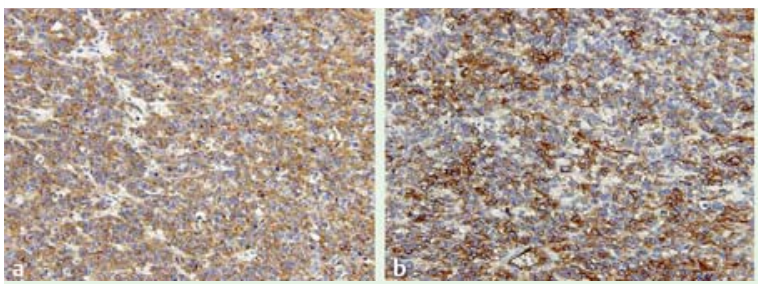

Fig. 5 Immunohistochemical staining showing a positive signal for a c-kit and b CD34 (magnification $\times 500$ ). c The labeling index (Ki-67) was 50\%-60\%.

ent case, on gastrointestinal endoscopy. Malignant potential of the tumor is predicted based on its size and mitotic activity [2]. Our patient presented with a peculiarly shaped GIST with high risk for malignancy that needed careful and longterm follow-up to monitor for signs of local recurrence or distant metastasis.

Endoscopy_UCTN_Code_CCL_1AB_2AC_3AB
K. Suzuki ${ }^{1}$, H. Takahashi ${ }^{1}$, Y. Abe ${ }^{1}$, M. Inamori', S. Watanabe', T. Koide', C. Tokoro' ${ }^{1}$, H. lida ${ }^{1}$, K. Hosono ${ }^{1}$,

H. Endo ${ }^{1}$, T. Shimamura ${ }^{1}$, N. Kobayashi ${ }^{1}$, H. Kirikoshi ${ }^{1}$, K. Kubota ${ }^{1}$, S. Saito',

H. Ono ${ }^{2}$, H. Akiyama ${ }^{2}$, T. Yazawa ${ }^{3}$,

A. Nakajima ${ }^{1}$

1 Gastroenterology Division, Yokohama City University School of Medicine, Yokohama, Japan

2 Division of Surgery, Yokohama City University School of Medicine, Yokohama, Japan

3 Department of Pathobiology, Yokohama City University Graduate School of Medicine, Yokohama, Japan

\section{References}

1 Shinomura Y, Kinoshita K, Tsutsui S et al. Pathophysiology, diagnosis, and treatment of gastrointestinal stromal tumors. J Gastroenterol 2005; 40: 775-780

2 Joensuu $H$. Gastrointestinal stromal tumor (GIST). Ann Oncol 2006; 17 Suppl 10: X280-X286

Bibliography

DOI $10.1055 / \mathrm{s}-0029-1243916$

Endoscopy 2010; 42: E65 - E66

(c) Georg Thieme Verlag KG Stuttgart · New York . ISSN 0013-726X

Corresponding author

H. Takahashi

Gastroenterology Division

Yokohama City University School of Medicine

3-9 Fuku-ura Kanazawa-ku

Yokohama 236-0004

Japan

Fax: +81-45-784-3546

hirokazu@med.yokohama-cu.ac.jp 